\title{
MIDIENDO LA HISTORIA MODERNA: \\ EL IMPACTO DE LA REVISTA «HISPANIA» \\ A TRAVÉS DE LAS REVISTAS UNIVERSITARIAS DE HISTORIA MODERNA ESPAÑOLAS
}

por

\author{
FRANCISCO TOSETE HERRANZ \\ Depto. de Historia Moderna, CSIC
}

RESUMEN: En el presente estudio se aborda el análisis del uso e impacto que Hispania tiene en las principales revistas universitarias españolas de Historia Moderna. El objetivo perseguido es conocer cuál es la utilización que los investigadores modernistas realizan de Hispania, determinando si la aparición en los últimos 30 años de otras revistas especializadas del área ha influido en su visibilidad.

Para ello, se ban analizado los números de dichas revistas publicados entre 1989 y 1998, estableciéndose el número de documentos de Historia Moderna citados y no citados de Hispania, la procedencia o distribución de las citas, las autocitas de autores, el indice de colaboración, el número de autores citados, quiénes son más citados y qué trabajos son los de mayor visibilidad, así como la antigüedad de los artículos citados.

PALABRAS Clave: Hispania. Historia Moderna. Revistas universitarias españolas. Bibliometría. Historiografía española.

ABSTRACT: The present article addresses the use and impact of the journal Hispania in the principal Spanish scholarly journals dedicated to modern history. The am is to discover what use modernists bave made of Hispania, and to determining whether its visibility bas been affected by the apprearance of other specialised journals over the last thirty years.

To this end, those issues of the scholarly journals published between 1989 and 1998 have been studied. This article will establish the number of texts in modern bistory from Hispania that bave been cited or not cited, the provenance and distribution of citations, self-citation by authors, the number of authors cited, those authors and texts most cited, and the age of the articles cited.

KEY WORDS: Hispania. Modern History. Spanish journals. Spanish Historiography. 


\section{INTRODUCCIÓN: UNA VISIÓN DE LA HISTORIOGRAFÍA ESPAÑOLA A TRAVÉS DE LA REVISTA HISPANIA}

Hispania es considerada hoy día una de las revistas de historia general más significativas constituyendo a lo largo de sus sesenta años de existencia, desde sus páginas, y a través de sus contenidos, un excelente punto de referencia para observar la evolución del panorama historiográfico español del último siglo ${ }^{1}$.

En 1907 se crea de forma paralela a la Universidad, la Junta de Ampliación de Estudios para apoyar y estimular la investigación española. Es entonces cuando aparece como un organismo dependiente de ella, el Centro de Estudios Históricos, institución que contribuiría al desarrollo científico de la Historia en nuestro país.

El período de la Guerra Civil supuso un auténtico retroceso para todos los ámbitos de la ciencia y la cultura y tuvo una importante repercusión en las que, hasta entonces, habían sido las únicas revistas históricas nacionales de carácter general que se publicaban con regularidad: el Boletín de la Real Academia de la Historia, aparecido en 1947, y el Anuario de Historia del Derecho Español, publicado desde 1934.

Al término de la guerra desapareció la Junta de Ampliación de Estudios surgiendo en su lugar el Consejo Superior de Investigaciones Científicas.

Cuatros años más tarde, en 1940, ve la luz el primer número de Hispania. Revista Española de Historia de carácter generalista, que se convertiría en el medio de expresión del recién creado Instituto de Historia de España Jerónimo Zurita. Este instituto sustituía, a su vez, al anterior Centro de Estudios Históricos.

Desde su origen y hasta la fecha, Hispania ha aparecido con regularidad hasta alcanzar en la actualidad los más de 200 números. Entre 1967 y 1983 se elaboraron un total de 10 números monográficos sobre diversas temáticas conocidos como los Cuadernos de Historia. Anexos de Hispania.

En 1985 desapareció el Instituto de Historia de España Jerónimo Zurita constituyéndose nuevamente en su lugar, el Centro de Estudios Históricos del CSIC, denominación que conservaría hasta 1998, momento en el que adoptaría su nombre actual de Instituto de Historia.

A finales de los años ochenta, en 1989, se celebraron unas jornadas para conmemorar el cincuentenario de la Revista de Indias e Hispania realizándose

\footnotetext{
* El autor quiere expresar su agradecimiento a la Dra. Inés Aragón González del Departamento de Biblioteconomía y Documentación de la Universidad Carlos III de Madrid, por la revisión del manuscrito y las numerosas sugerencias y aportaciones realizadas, a la Dra. Isabel Gómez Caridad, del Departamento de Bibliometría y Análisis Documental del CINDOC, CSIC, por la valiosa ayuda totalmente desinteresada para la realización del artículo y, especialmente, al Dr. Francisco Fernández Izquierdo, del Departamento de Historia Moderna del Instituto de Historia, CSIC, cuyo apoyo ha sido decisivo para la buena conclusión de este trabajo.

1 Para una visión más detallada de la trayectoria de la revista puede consultarse ESTEPA, C., Las revistas de Historia en España: El ejemplo de Hispania.
} 
unas actas que fueron recogidas de forma íntegra en los números 175 y 176 del año 1990. Los artículos presentados ofrecían una aproximación acerca de la trayectoria de los contenidos de Hispania y de la historiografía española a lo largo de su existencia.

En estas jornadas Celso Almuiña y colaboradores ${ }^{2}$ presentaron un artículo en el que realizaban un análisis cuantitativo sobre la evolución de la revista y sus contenidos, y del cual podía deducirse la gran presencia que, desde sus comienzos, había tenido la Historia Moderna en sus páginas.

A partir de 1995 Hispania pasó a incorporar una sección de dossieres o trabajos encargados a propósito de un tema, coordinados por un especialista. Se mantenían, asimismo, las secciones de «Artículos», «Notas y Ensayos», «Textos y Documentos» y una última de «Bibliografía y Recensiones». En este año la revista aumentó su número de páginas centrándose en una línea editorial que potenciaba la presencia de estudios o números monográficos ${ }^{3}$.

En la actualidad, Hispania es una publicación cuatrimestral que tiene una extensión total de 1.200 páginas distribuidas en tres números anuales que aparecen respectivamente en abril, agosto y diciembre. Hoy día es considerada, entre otras, una de las publicaciones más importantes del panorama historiográfico español, tanto por el amplio período de existencia como por el número de trabajos y los historiadores que publican en ella. Se encuentra recogida, además, desde hace años, en diversos repertorios bibliográficos internacionales como los Índices del CINDOC, el Historical Abstract o el Periodical Current Index.

\section{OBJETIVOS}

Tal y como se pone de manifiesto en el estudio llevado a cabo por Celso Almuiña Fernández y colaboradores, el área de Historia Moderna es la que, desde sus inicios, ha estado mejor representada en la revista en cuanto a número de artículos aparecidos, llegando a suponer más del $40 \%$ del total de los publicados.

Uno de los objetivos más significativos del presente estudio, es determinar cuál es el impacto que los artículos del área, aparecidos en Hispania, han tenido en las principales revistas universitarias españolas de Historia Moderna, estableciendo el uso que los historiadores realizan de los mismos.

Con ello, se pretende conocer la visibilidad de la revista en el ámbito de la comunidad científica de modernistas, además de confirmar su validez como instrumento para medir el panorama historiográfico nacional tanto retrospectivamente como en la actualidad.

\footnotetext{
2 Almuiña Fernández, C.: et. al. Hispania, revista de Historia (1940-1989). Análisis y evolución de contenidos.

3 ESTEPA, C.: op. cit. p. 307
} 
A partir del estudio de la tipología de los documentos citados publicados en Hispania se puede determinar cuál o cuáles son las secciones más relevantes desde el punto de vista científico, en cuanto al área de Historia Moderna se refiere.

Asimismo, el análisis de los artículos citados permitirá ofrecer una aproximación acerca de las temáticas tratadas identificando aquellas en las que los autores inciden más.

Otro aspecto significativo que se pretende delimitar es el período en el que son publicados los trabajos de historia moderna aparecidos en Hispania que mayor impacto han tenido entre la comunidad de modernistas determinando, además, el tiempo medio transcurrido desde que un artículo es publicado hasta que recibe una cita.

Por último, se estudian distintos indicadores como las autocitas, el nivel de colaboración, o la distribución de las citas entre los autores, con el fin de ofrecer una visión general de los hábitos de trabajo y citación de los historiadores modernistas españoles y de las instituciones en las que trabajan.

\section{METOdología:}

El presente estudio se limita a la última década (1989-1998) debido a que este período constituye, aproximadamente, la mitad del tiempo durante el cual llevan publicándose las revistas de Historia Moderna de ámbito universitario a excepción de Chronica Nova y Estudis, aparecidas en 1968 y 1972 respectivamente. Todas las demás revistas comienzan a publicarse con posterioridad, ofreciéndose así, una visión lo suficientemente significativa de la utilización e impacto de Hispania en los últimos años.

A la hora de realizar la selección se ha prescindido de aquéllas revistas de carácter generalista, seleccionando las específicas del área de Moderna.

Las revistas que se han escogido para el presente estudio han sido:

- Cbronica Nova de la Universidad de Granada (1968)

- Cuadernos de Historia Moderna de la Universidad Complutense de Madrid (1980)

- Estudis. Revista de Historia Moderna de la Universidad de Valencia (1972)

- Hispania. Revista Española de Historia del Instituto de Historia del CSIC (1940)

- Manuscrits. Revista d' Historia Moderna de la Universidad Autónoma de Barcelona (1986)

- Obradoiro de Historia Moderna de la Universidad de Santiago de Compostela (1992)

- Pedralbes. Revista d' Historia Moderna de la Universidad de Barcelona (1981) 
- Revista de Historia Moderna. Anales de la Universidad de Alicante (1983)

- Stvdia Histórica. Historia Moderna de la Universidad de Salamanca (1983)

La propia revista Hispania se ha incluido, con el fin de poder observar y determinar cuáles eran los hábitos de citación de los autores que publicaban en ella y de los trabajos aparecidos en la misma.

Los datos estudiados se han obtenido a partir de la revisión completa de todas las secciones de cada revista, exceptuando aquéllas en las que se daba noticia de eventos tales como la celebración o informe de congresos, conferencias de clausura, mesas redondas, coloquios, o en las que se presentaban resúmenes de tesis doctorales, tesinas, recensiones, etc. En el caso de Hispania se seleccionaron los artículos de las secciones citadas cuya temática se enmarcaba en el período transcurrido desde el establecimiento del reinado de los Reyes Católicos hasta la Guerra de la Independencia.

En ocasiones fue difícil extraer las referencias, dado que podían aparecer insertas en el texto de los artículos, de forma incompleta, o anejas como bibliografía final adjunta a los mismos, siendo complicado determinar su número real.

En concreto, para este trabajo, únicamente se tuvieron en cuenta aquellas referencias completas de los trabajos publicados tanto en Hispania como en los números monográficos conocidos como los Anexos aparecidos entre 1967 y 1983.

En cuanto a los artículos citados, los datos se obtuvieron de la versión disponible en línea de la base de datos de la revista Hispania ${ }^{4}$.

De cada uno de los artículos de Hispania se recogió el autor, título, número y volumen de publicación, año y la extensión del trabajo en número de páginas.

Asimismo, de los artículos con referencias a Hispania, se obtuvo el autor, título, institución de trabajo, en caso de existir, número o volumen de publicación, año en que aparecía, número de páginas del artículo y número total de referencias realizadas por el autor en él.

A partir de éstos se determinó:

- La tipología y temática de los documentos de Hispania citados

- El número de los mismos citados y no citados

- La procedencia de las citas

- El número de documentos citados por decenios

- Las autocitas de autores, tanto desde la propia Hispania como desde otras revistas

- El índice de colaboración de los autores de los documentos citados

- Los autores citados y la distribución de citas entre ellos

- Los autores más citados

- Los trabajos más citados y su distribución por décadas

- La antigüedad de los trabajos citados

4 Puede ser consultada en http://www.modernal.ih.csic.es/hispania/ 
A la hora de abordar la temática de los artículos se recurrió a especialistas en Historia Moderna para ofrecer el mayor rigor posible. La clasificación establecida partía de la realizada en su trabajo por Almuiña y colaboradores aunque se ampliaron algunas categorías para ser más significativas.

En su conjunto, estos indicadores ofrecen una idea, tanto del uso que se realiza de los trabajos de Historia Moderna aparecidos en Hispania, como de los hábitos de citación y trabajo de los modernistas españoles a partir de los estudios publicados durante la última década.

\section{Resultados}

\subsection{Número de trabajos publicados en las distintas revistas analizadas}

TABLA 1. Distribución del número de trabajos de Historia Moderna publicados en Hispania (1989-1998)

\begin{tabular}{|l|c|c|c|c|c|c|c|c|c|c|c|}
\hline REVISTAS & 1989 & 1990 & 1991 & 1992 & 1993 & 1994 & 1995 & 1996 & 1997 & 1998 & TOTAL: \\
\hline Hispania & 11 & 24 & 9 & 15 & 11 & 11 & 8 & 12 & 7 & 14 & 122 \\
\hline
\end{tabular}

TABLA 2. Distribución del número de trabajos de Historia Moderna publicados en las distintas revistas analizadas (1989-1998)

\begin{tabular}{|c|c|c|c|c|c|c|c|c|c|c|c|}
\hline REVISTAS & 1989 & 1990 & 1991 & 1992 & 1993 & 1994 & 1995 & 1996 & 1997 & 1998 & TOTAL: \\
\hline Pedralbes & 6 & 0 & 13 & 11 & 124 & 9 & 12 & 6 & 11 & 90 & 290 \\
\hline Chronica Nov & 19 & 18 & 18 & 16 & $9^{*}$ & $9^{*}$ & 16 & 16 & 25 & 20 & 166 \\
\hline $\begin{array}{l}\text { Cuader } \\
\text { Moder }\end{array}$ & $5^{*}$ & $5^{*}$ & 13 & 12 & 15 & $17^{\circ}$ & 21 & 22 & 21 & 21 & 152 \\
\hline Manuscrits & - & 14 & 14 & 16 & 11 & 14 & 14 & 16 & 23 & 16 & 138 \\
\hline Studia Histórica & 5 & 15 & 12 & $5^{*}$ & $4^{*}$ & 13 & 12 & 23 & 22 & 12 & 123 \\
\hline Estudis & 10 & 8 & 5 & 11 & 9 & 14 & 11 & 10 & 12 & 23 & 113 \\
\hline $\begin{array}{l}\text { Revis } \\
\text { Mod }\end{array}$ & $8^{*}$ & $7^{*}$ & 11 & 13 & 8 & $8^{*}$ & $7^{*}$ & 20 & 18 & 18 & 118 \\
\hline $\begin{array}{l}\text { Obradoiro } \\
\text { Moderna }\end{array}$ & - & - & - & 9 & 8 & 7 & 9 & 10 & 7 & 7 & 57 \\
\hline TOTAL: & 53 & 75 & 86 & 93 & 188 & 91 & 102 & 123 & 139 & 207 & 1157 \\
\hline
\end{tabular}

Un "*" al lado de cualquiera de los números indica que los trabajos publicados han sido distribuidos equitativamente entre los años correspondientes debido a que sólo apareció un número de la revista en esos dos años. Un "-" indica que en ese año no se publicó ningún número de la misma. La revista Obradoiro de Historia Moderna comenzó a publicarse en 1992. 
Entre 1989 y 1998 se publicaron 1279 trabajos de Historia Moderna en las revistas analizadas (incluyendo artículos o estudios críticos, notas bibliográficas, ensayos, bibliografía histórica, textos y documentos o miscelánea) que constituían el total de la población examinada en el presente estudio. De ellos, 122 $(9,5 \%)$ aparecieron en la revista Hispania (tabla 1) y 1157 en el resto de revistas estudiadas. En la tabla 2 se observa cómo Pedralbes fue la revista en la que mayor número de trabajos se recogieron debido a que los números aparecidos en 1993 y 1998 correspondían a las actas íntegras del III y IV congresos de Historia Moderna de Cataluña. Esto supone que, en ese mismo período, por término medio, fueron publicados en el conjunto de las revistas analizadas 142,1 artículos.

TABLA 3. Número de artículos de Historia Moderna publicados en Hispania (1940-1998)

\begin{tabular}{|l|c|c|c|}
\hline DECENIOS & No ART. PUB. HISPANIA $^{\text {No }}$ & $\begin{array}{c}\text { N }^{\circ} \text { ART. PUB. } \\
\text { ANEXOS HISPANIA }\end{array}$ & TOTAL ART. PUB. \\
\hline $1940-1949$ & 53 & - & 53 \\
$1950-1959$ & 66 & - & 66 \\
$1960-1969$ & 54 & 23 & 77 \\
$1970-1979$ & 41 & 31 & 72 \\
$1980-1989$ & 59 & 7 & 66 \\
$1990-1998$ & 100 & - & 100 \\
\hline TOTAL: & 373 & 61 & 434 \\
\hline
\end{tabular}

En la tabla 3 se recogen, únicamente, los trabajos de Historia Moderna de Hispania en la sección artículos/estudios. Desde sus comienzos hasta finales de la década de los noventa aparecieron en ella un total de 434 artículos, de los cuales 61 fueron publicados entre 1967 y 1983 en los Cuadernos de Historia. Anexos de Hispania y 373 en la propia revista Hispania. En los últimos 8 años la presencia de la Historia Moderna en las páginas de Hispania aumentó de forma considerable hasta llegar a los 100 artículos publicados. En este período aparecieron, asimismo, los dos números monográficos de 1990 en los que se recogían íntegramente las actas sobre la celebración del cincuentenario de la revista.

En los sesenta años de existencia se publicaron por término medio en Hispania y Anexos 72,3 artículos de Historia Moderna cada década. 


\subsection{Tipología y temática de los trabajos de Hispania citados}

TABLA 4. Tipología de los trabajos de Historia Moderna publicados en Hispania citados

\begin{tabular}{|l|c|c|}
\hline TIPO & N $^{\circ}$ CIT. HECHAS A TRAB. & $\%$ \\
\hline DE HISPANIA Y ANEXOS & 340 & 92,39 \\
Trtículos & 14 & 3,80 \\
Rextos y documas & 10 & 2,72 \\
Miscelánea & 2 & 0,54 \\
Bibliografía histórica & 1 & 0,27 \\
Estudios críticos & 1 & 0,27 \\
\hline Total: & 368 & 100 \\
\hline
\end{tabular}

Del análisis de la tipología de los trabajos citados de Historia Moderna se observaba cómo la mayor parte, de las 368 citas realizadas, el 92,39\%, correspondían a artículos/estudios siendo ésta, la sección más representativa y con mayor contenido científico de la revista. Los trabajos de otras secciones eran escasamente citados, suponiendo menos del $8 \%$ de las citas totales recogidas.

TABLA 5. Temática de los artículos de Historia Moderna publicados en Hispania citados

\begin{tabular}{|c|c|c|c|}
\hline TEMÁTICA & $\begin{array}{l}N^{\circ} \text { CIT. HECHAS A ART. DE } \\
\text { HISPANIA Y ANEXOS }\end{array}$ & $\%$ & $\%$ ACUM. \\
\hline Instituciones & 65 & 19,12 & 19,12 \\
\hline $\begin{array}{l}\text { Grupos sociales, mentalidad, conflictividad } \\
\text { social }\end{array}$ & 60 & 17,65 & 36,76 \\
\hline Aspectos económicos & 58 & 17,06 & 53,82 \\
\hline Demografía, poblamiento, alimentación & 52 & 15,29 & 69,12 \\
\hline Biografías & 21 & 6,18 & 75,29 \\
\hline Relaciones internacionales & 21 & 6,18 & 81,47 \\
\hline Historia política & 17 & 5,00 & 86,47 \\
\hline Teoría y método de la historia, historiografía & 16 & 4,71 & 91,18 \\
\hline Fuentes & 11 & 3,24 & 94,41 \\
\hline Cultura y pensamiento & 10 & 2,94 & 97,35 \\
\hline Religión & 9 & 2,65 & 100 \\
\hline TOTAL: & 340 & 100 & \\
\hline
\end{tabular}

Hispania, LXII/1, núm. 210 (2002) 41-64 
El aspecto temático se abordó desde una perspectiva amplia con el fin de poder contar con una panóramica general de las materias. Aunque ciertamente podría haberse realizado una clasificación exhaustiva y en profundidad, ello hubiera conllevado la pérdida de la visión de conjunto.

De esta forma, la presente clasificación no pretende ser taxativa sino ofrecer una orientación sobre las principales materias tratadas por los autores de los artículos citados teniendo en cuenta, además que, aunque determinados trabajos podían haberse encuadrado en más de una temática, únicamente se les asignó aquélla que resultaba más significativa.

Casi una quinta parte de los artículos citados versaban sobre las instituciones o su historia $(19,12 \%)$ seguidos de los relativos a la sociedad o los aspectos sociales $(17,65 \%)$. Los estudios sobre la economía eran, asimismo, ampliamente citados $(17,06 \%)$ junto a los que trataban sobre la población, demografía o la alimentación en el período moderno $(15,29 \%)$. Estos cuatro grupos temáticos constituían cerca del $70 \%$ del total de los artículos citados.

En menor medida se daban los estudios biográficos y sobre relaciones internacionales $(6,18 \%$ ambas) e historia política $(5 \%)$ mientras que el resto de las temáticas, fuentes, cultura, pensamiento y religión, mantenían un porcentaje inferior al $5 \%$, respectivamente, de los artículos citados.

\subsection{Artículos de Hispania citados}

TABLA 6. Número de artículos publicados de Historia Moderna en Cuadernos de Historia. Anexos de Hispania y número de artículos citados

\begin{tabular}{|l|c|c|c|c|c|}
\hline DECENIOS & N $^{\circ}$ ART. PUB. & $N^{\circ}$ ART. PUB. CIT. & $\begin{array}{c}\mathrm{N}^{\circ} \text { CIT. HECHAS A } \\
\text { ART. PUB. ANEXOS }\end{array}$ & $\begin{array}{c}\text { \% ART CIT. PUB. } \\
\text { ANEXOS }\end{array}$ & $\begin{array}{c}\text { \% ART NO CIT. } \\
\text { PUB. ANEXOS }\end{array}$ \\
\hline $1940-1949$ & - & - & - & - & - \\
$1950-1959$ & - & - & - & - & - \\
$1960-1969$ & 23 & 7 & 13 & 30,43 & 69,57 \\
$1970-1979$ & 31 & 8 & 8 & 25,81 & 74,19 \\
$1980-1989$ & 7 & 1 & 1 & 14,29 & 85,71 \\
$1990-1998$ & - & - & - & - & - \\
\hline TOTAL: & 61 & 16 & 22 & 26,23 & 73,77 \\
\hline
\end{tabular}

Desde 1967 y hasta 1983 se publicaron 10 números monográficos complementarios a Hispania conocidos como los Cuadernos de Historia. Anexos de Hispania. En ellos aparecieron 61 artículos de Historia Moderna de los cuales fueron citados el 26,23\%, un total de 16 artículos (tabla 6) recibiendo cada uno de ellos 1,37 citas por término medio. 45 artículos $(73,77 \%)$ no habían sido citados en el conjunto de revistas analizadas. 
TABLA 7. Número de artículos publicados de Historia Moderna en Hispania y número de artículos citados

\begin{tabular}{|l|r|c|c|c|c|}
\hline DECENIOS & $\begin{array}{c}\mathbf{N}^{\circ} \text { ART. } \\
\text { PUB. }\end{array}$ & N $^{\circ}$ ART. PUB. CIT. & $\begin{array}{c}\mathbf{N}^{\circ} \text { CIT. HECHAS A } \\
\text { ART. PUB. HISPANIA }\end{array}$ & $\begin{array}{c}\text { \% ART CIT. PUB. } \\
\text { EN HISPANIA }\end{array}$ & $\begin{array}{c}\text { \% ART NO CIT. } \\
\text { PUB. EN HISPANIA }\end{array}$ \\
\hline $1940-1949$ & 53 & 20 & 23 & 37,74 & 62,26 \\
$1950-1959$ & 66 & 27 & 43 & 40,91 & 59,09 \\
$1960-1969$ & 54 & 32 & 57 & 59,26 & 40,74 \\
$1970-1979$ & 41 & 30 & 48 & 73,17 & 26,83 \\
$1980-1989$ & 59 & 40 & 79 & 67,80 & 32,20 \\
$1990-1998$ & 100 & 46 & 68 & 46,00 & 54,00 \\
\hline TOTAL: & 373 & 195 & 318 & 52,28 & 47,72 \\
\hline
\end{tabular}

En Hispania fueron publicados, desde sus inicios, 373 artículos de Historia Moderna, de los cuales se citaron 195, algo más de la mitad 52,28\%. Este número de artículos recibió un total de 318 citas, dado que un mismo artículo podía ser citado más de una vez (tabla 7 ). 178 artículos $(47,72 \%)$ no recibieron ninguna cita y por término medio cada artículo fue citado 1,63 veces.

El período en el que se daba mayor número de artículos citados frente a los publicados, era la década de los años setenta. Este período fue en el que menor número de artículos se publicaron, un total de 41 , y mayor número de ellos se , citaron, $30(73,17 \%)$.

Aunque cada década se citaban más artículos de Historia Moderna esto no era significativo dado que, desde 1970 hasta la actualidad, se observaba un descenso del nivel de artículos citados respecto de los publicados (tabla 7). Esta disminución era particularmente acusada en los últimos nueve años, llegando a suponer hasta un $21,8 \%$ menos que la década anterior.

Una de las razones que ayuda a explicar este fenómeno es la aparición, en el ámbito universitario, de las revistas especializadas de Historia Moderna a finales de los años setenta, existiendo mayor diversidad de medios en los que publicar. Por otro lado, estos nueve años, son el período en el que mayor número de artículos de Historia Moderna se publicaron en Hispania, suponiendo un incremento respecto del anterior del 69,5\%. 


\subsection{Procedencia de las citas}

\subsubsection{Distribución por revistas de las citas realizadas a Hispania}

TABLA 8. citas realizadas a trabajos de Historia Moderna de Hispania desde otros trabajos publicados en la propia revista

\begin{tabular}{|l|c|}
\hline REVISTA & $\begin{array}{r}N^{\circ} \text { DE CITAS HECHAS A TRAB. PUB. } \\
\text { EN HISPANIA Y ANEXOS }\end{array}$ \\
\hline Hispania & 130 \\
\hline
\end{tabular}

TABLA 9. Distribución por revistas de las citas realizadas a trabajos de Historia Moderna publicados en Hispania

\begin{tabular}{|l|c|c|c|}
\hline REVISTA & $\begin{array}{c}\text { N }^{\circ} \text { DE CITAS HECHAS A TRAB. PUB. } \\
\text { EN HISPANIA Y ANEXOS }\end{array}$ & $\%$ & $\%$ ACUM. \\
\hline Cuadernos de Historia Moderna & 74 & 31,09 & 31,09 \\
\hline Studia Histórica & 44 & 18,49 & 49,58 \\
\hline Chronica Nova & 34 & 14,29 & 63,87 \\
\hline Manuscrits & 28 & 11,76 & 75,63 \\
\hline Pedralbes & 22 & 9,24 & 84,87 \\
\hline Obradoiro de Historia Moderna & 14 & 5,88 & 90,76 \\
\hline Revista de Historia Moderna & 13 & 5,46 & 96,22 \\
\hline Estudis & 9 & 3,78 & 100 \\
\hline Total: & 238 & 100 & \\
\hline
\end{tabular}

Del total de las 368 citas realizadas a trabajos de Historia Moderna publicados en las páginas de Hispania, 130 se hicieron desde otros trabajos que aparecieron en ella con posterioridad (tabla 8).

De las 238 citas restantes (tabla 9), el 31,09\% se había realizado desde Cuadernos de Historia Moderna. Studia Histórica era la segunda revista de las analizadas desde la que se citaron más trabajos de Hispania, 18,49\%, seguida de Chronica Nova, 11,76\% y Pedralbes, 9,24\%. Desde las páginas de Obradoiro de Historia Moderna se realizaron algo menos del $6 \%$ de las citas, porcentaje similar al de la Revista de Historia Moderna, 5,46\%. Estudis era la publicación en la que que los autores citaban menos trabajos de Hispania, tan sólo un 3,78\%. 


\subsubsection{Distribución cronológica de los trabajos citados}

Tabla 10. Distribución cronológica de los trabajos citados

\begin{tabular}{|l|c|r|c|}
\hline DECENIOS & $\begin{array}{c}\text { N }^{\circ} \text { DE CITAS HECHAS A TRAB. } \\
\text { PUB. EN HISPANIA Y ANEXOS }\end{array}$ & $\%$ & $\%$ ACUM. \\
\hline $1940-1949$ & 24 & 6,52 & 6,52 \\
$1950-1959$ & 44 & 11,96 & 18,48 \\
$1960-1969$ & 78 & 21,20 & 39,67 \\
$1970-1979$ & 62 & 16,85 & 56,52 \\
$1980-1989$ & 88 & 23,91 & 80,43 \\
$1990-1998$ & 72 & 19,57 & 100 \\
\hline TOTAL: & 368 & 100 & \\
\hline
\end{tabular}

El $18,48 \%$ de las citas encontradas correspondían a trabajos publicados en los primeros veinte años de existencia de la revista. Los aparecidos entre 1960 y 1979 recibieron más del doble de las realizadas en el anterior período $(38,05 \%)$.

Una gran parte de las citas $(43,48 \%)$ eran de artículos y trabajos publicados desde 1980 hasta la actualidad. Puede observarse, además, cómo las citas se distribuían equitativamente en los cuarenta últimos años situándose en torno al $20 \%$ cada década.

\subsubsection{Distribución de las autocitas de autor}

Muchos de los autores que publicaban en las revistas analizadas referenciaban trabajos suyos aparecidos con anterioridad en Hispania. Es interesante conocer en qué medida estos autores se autocitaban, con el fin de determinar, entre otros aspectos, los hábitos de investigación de los modernistas españoles, y dónde y cómo publicaban los resultados derivados de la continuidad de sus líneas de investigación.

TABLA 11. Nivel de autocitación de los autores desde trabajos de la propia revista Hispania en el período estudiado

\begin{tabular}{|c|c|c|c|}
\hline Revistas & $\mathrm{N}^{\circ}$ AUtOCitas en HisPania & $\begin{array}{l}\mathrm{N}^{\circ} \text { DE CITAS HECHAS A } \\
\text { TRAB. PUB. EN HISPANIA }\end{array}$ & $\%$ AUTOC. EN HisPania \\
\hline Hispania & 9 & 130 & 6,92 \\
\hline
\end{tabular}


Tabla 12. Nivel de autocitación de los autores desde las revistas analizadas en el período estudiado

\begin{tabular}{|l|c|c|c|}
\hline REVISTAS & $\begin{array}{c}\text { No }^{\circ} \text { AUTOCITAS POR } \\
\text { REV. }\end{array}$ & $\begin{array}{c}\text { N }^{\circ} \text { DE CITAS HECHAS A } \\
\text { TRAB. DE HISPANIA }\end{array}$ & $\begin{array}{c}\% \text { AUTOC. POR } \\
\text { REV. }\end{array}$ \\
\hline Pedralbes & 4 & 22 & 18,18 \\
Manuscrits & 4 & 28 & 14,29 \\
Studia Histórica & 6 & 44 & 13,64 \\
Chronica Nova & 4 & 34 & 11,76 \\
Cuadernos de Historia Moderna & 4 & 74 & 5,41 \\
\hline TOTAL: & 22 & 202 & $\mathbf{1 0 , 8 9}$ \\
\hline
\end{tabular}

De las 36 citas de trabajos de Historia Moderna publicados en Hispania que se habían realizado desde las páginas de Estudis, Obradoiro de Historia Moderna y Revista de Historia Moderna (tabla 9) ninguna de ellas era una autocita. Estas revistas y sus correspondientes citas no se recogieron, por lo tanto, en la tabla 12.

El porcentaje de autocitas que se daba en Hispania era relativamente bajo, siendo inferior al $7 \%$, como puede observarse en la tabla 11 . En cuanto al resto de publicaciones el índice de autocitación tampoco era excesivamente elevado $(10,89 \%)$. Pedralbes era la publicación en la que mayor nivel de autocitación se daba $(18,18 \%)$.

4.5. Índice de colaboración de los trabajos citados

TABLA 13. Índice de colaboración

\begin{tabular}{|c|c|c|c|}
\hline AUTORES POR TRAB. & No DE TRAB. CIT. & TOTAL AUT. EN TRAB. CIT. & $\%$ \\
\hline 2 & 24 & 48 & 12,24 \\
1 & 344 & 344 & 87,76 \\
\hline & 368 & 392 & 100 \\
\hline
\end{tabular}

De la tabla 13 se deduce que la mayor parte de los autores de los trabajos citados de Hispania escribía habitualmente en solitario, $87,76 \%$, frente al $12,24 \%$ que lo hacía en colaboración. El índice de colaboración o la media de autores que firmaban cada trabajo citado es 1,06 . Se observa, por lo tanto, cómo en el ámbito español y en el período analizado, el trabajo de investigación histórica sigue siendo una labor que se desarrolla y es abordada, fundamentalmente, de forma individual. 


\subsection{Número de autores citados}

La distribución del número de citas que han recibido los trabajos aparecidos en Hispania por autores permite conocer el grado de dispersión de las mismas y el porcentaje de autores más productivos que concentran el mayor número de citas.

TABLA 14. Distribución del número de citas por autores de los trabajos de Historia Moderna publicados en Hispania

\begin{tabular}{|l|c|c|c|c|c|c|}
\hline $\begin{array}{l}\text { CitAS POR } \\
\text { AUTOR }\end{array}$ & $\begin{array}{c}\text { AUT. CON } \\
\text { N CIT. }\end{array}$ & $\begin{array}{c}\text { TOTAl AUT. } \\
\text { EN TRAB. CIT. }\end{array}$ & $\begin{array}{c}\text { ACUM. AUT. } \\
\text { CON N CIT. }\end{array}$ & $\begin{array}{c}\text { ACUM. TOT. AUT. } \\
\text { EN TRAB. CIT. }\end{array}$ & $\begin{array}{c}\text { \% ACUM. AUT. } \\
\text { CON N CIT. }\end{array}$ & $\begin{array}{c}\text { \% ACUM. TOT. } \\
\text { AUT. EN TRAB. CIT. }\end{array}$ \\
\hline 15 & 1 & 15 & 1 & 15 & 0,57 & 3,83 \\
14 & 1 & 14 & 2 & 29 & 1,14 & 7,40 \\
13 & 1 & 13 & 3 & 42 & 1,71 & 10,71 \\
12 & 2 & 24 & 5 & 66 & 2,86 & 16,84 \\
9 & 1 & 9 & 6 & 75 & 3,43 & 19,13 \\
8 & 3 & 24 & 9 & 99 & 5,14 & 25,26 \\
7 & 1 & 7 & 10 & 106 & 5,71 & 27,04 \\
6 & 3 & 18 & 13 & 124 & 7,43 & 31,63 \\
5 & 7 & 35 & 20 & 159 & 11,43 & 40,56 \\
4 & 6 & 24 & 26 & 183 & 14,86 & 46,68 \\
3 & 9 & 27 & 35 & 210 & 20,00 & 53,57 \\
2 & 42 & 84 & 77 & 294 & 44,00 & 75,00 \\
1 & 98 & 98 & 175 & 392 & 100 & 100 \\
\hline Total: & 175 & 392 & & & & \\
\hline
\end{tabular}

El número de autores totales firmantes de los trabajos citados de Hispania eran 392. Estos se correspondían con 175 autores únicos (un mismo autor podía recibir más de una cita). El 2,86\% de los autores únicos recibían 12 o más citas y el $14,86 \%$ eran citados cuatro o más veces (tabla 14). Más de la mitad de los autores, 98 (56\%) eran citados solamente una vez y 42 de ellos (24\%) lo eran dos o más veces. 


\subsection{Autores más citados}

TABLA 15. Autores con mayor número de citas en el período estudiado

\begin{tabular}{|c|c|c|c|c|}
\hline AUTOR & INSTITUCIÓN DE TRABAJO & ESPECIALIDAD & $\begin{array}{c}\mathrm{N}^{\circ} \text { DE CITAS } \\
\text { RECIBIDAS }\end{array}$ & $\begin{array}{c}\mathrm{N}^{\circ} \\
\text { AUTOCIT. }\end{array}$ \\
\hline Ladero Quesada, Miguel Ángel & $\begin{array}{l}\text { Univ. Complutense de } \\
\text { Madrid }\end{array}$ & Medieval & 15 & 0 \\
\hline Gutiérrez Nieto, Juan Ignacio & CSIC* & Moderna & 14 & 0 \\
\hline Moxó, Salvador de & $\begin{array}{l}\text { Univ. Complutense de } \\
\text { Madrid }\end{array}$ & Medieval & 13 & $\theta$ \\
\hline Domínguez Ortiz, Antonio & $\begin{array}{l}\text { Real Academia de la } \\
\text { Historia }\end{array}$ & Moderna & 12 & 0 \\
\hline Martínez Millán, José & $\begin{array}{l}\text { Univ. Autónoma de } \mathrm{Ma}- \\
\text { drid }\end{array}$ & Moderna & 12 & 9 \\
\hline Cepeda Adán, José & $\begin{array}{l}\text { Univ. Complutense de } \\
\text { Madrid }\end{array}$ & Moderna & 9 & 0 \\
\hline Kamen, Henry & $\begin{array}{l}\text { Diversas universidades } \\
\text { británicas** }\end{array}$ & Moderna & 8 & 0 \\
\hline Mercader Riba, Juan & $\begin{array}{l}\text { Instituto de Historia Jeró- } \\
\text { nimo Zurita. CSIC }\end{array}$ & $\begin{array}{l}\text { Moderna y Con- } \\
\text { temporánea }\end{array}$ & 8 & 0 \\
\hline Zudaire Huarte, Emilio & O.F.M. cap. (Franciscanos) & Moderna & 8 & 0 \\
\hline $\begin{array}{l}\text { Echevarría Bacigalupe, Miguel } \\
\text { Ángel }\end{array}$ & Univ. del País Vasco & Moderna & 7 & 5 \\
\hline Espadas Burgos, Manuel & CSIC & Contemporánea & 6 & 0 \\
\hline Martín Galán, Manuel & $\begin{array}{l}\text { Univ. Complutense de } \\
\text { Madrid }\end{array}$ & Moderna & 6 & 0 \\
\hline Ruiz Martín, Felipe & $\begin{array}{l}\text { Univ, Autónoma de Ma- } \\
\text { drid }\end{array}$ & Moderna & 6 & 0 \\
\hline $\begin{array}{l}\text { Álvarez Coca González, María } \\
\text { Jesús }\end{array}$ & Archivo Histórico Nacional & Archivística & 5 & 3 \\
\hline Atienza, Ignacio & $\begin{array}{l}\text { Univ. Autónoma de Ma- } \\
\text { drid }\end{array}$ & Moderna & 5 & 1 \\
\hline Diago Hernando, Máximo & CSIC & Medieval & 5 & 2 \\
\hline León Sanz, Virginia & $\begin{array}{l}\text { Univ. Complutense de } \\
\text { Madrid }\end{array}$ & Moderna & 5 & 3 \\
\hline Luxán Meléndez, Santiago de & Profesor E.S. Las Palmas & Moderna & 5 & 0 \\
\hline Peset, José Luis & Instituto de Historia. CSIC & $\begin{array}{l}\text { Historia de la } \\
\text { Ciencia }\end{array}$ & 5 & 0 \\
\hline Reglá, Juan & Univ. de Valencia & Moderna & 5 & 0 \\
\hline
\end{tabular}

* Los artículos citados corresponden al período en que trabajó en el CSIC. En la actualidad su institución de trabajo es la Universidad Complutense de Madrid.

** Actualmente trabaja en el CSIC en la Institución Milá i Fontanals de Barcelona.

En la tabla 15 se recogen los autores con cinco o más citas. El $75 \%$ de ellos trabajaban en centros u organismos de investigación de Madrid. El resto de los investigadores desarrollaban su actividad en distintas universidades y organismos españoles. Es interesante señalar cómo 6 de estos autores (30\%) no eran modernistas, 3 de ellos son especialistas en Historia Medieval y los restantes en Historia Contemporánea, Historia de la Ciencia y Archivística. 


\subsection{Artículos más citados}

TABLA 16. Artículos más citados en el período estudiado

\begin{tabular}{|c|c|c|c|c|}
\hline AUTOR & ARTíCULO & $\begin{array}{c}\text { AÑO DE } \\
\text { PUBLICACIÓN }\end{array}$ & $\begin{array}{l}\mathrm{N}^{\circ} \text { CIT. } \\
\text { REC. }\end{array}$ & AUTOCIT \\
\hline Kamen, Henry & $\begin{array}{l}\text { El establecimiento de los intendentes en } \\
\text { la administración española }\end{array}$ & 1964 & 8 & 0 \\
\hline $\begin{array}{l}\text { Ladero Quesada, Miguel } \\
\text { Angel }\end{array}$ & $\begin{array}{l}\text { La repoblación del reino de Granada } \\
\text { anterior al año } 1500\end{array}$ & 1968 & 7 & 0 \\
\hline Martín Galán, Manuel & $\begin{array}{l}\text { Fuentes y métodos para el estudio de la } \\
\text { Demografía Histórica castellana durante } \\
\text { la Edad Moderna }\end{array}$ & 1981 & 6 & 0 \\
\hline Atienza, Ignacio & $\begin{array}{l}\text { La "quiebra" de la nobleza castellana en } \\
\text { el siglo XVII. Autoridad real y poder } \\
\text { señorial: el secuestro de los bienes de la } \\
\text { Casa de Osuna }\end{array}$ & 1984 & 5 & 1 \\
\hline $\begin{array}{l}\text { Echevarría Bacigalupe, } \\
\text { Miguel Ángel }\end{array}$ & $\begin{array}{l}\text { Relaciones económicas y fiscales en la } \\
\text { monarquía hispánica, siglos XVI y XVII }\end{array}$ & 1991 & 5 & 3 \\
\hline León Sanz, Virginia & $\begin{array}{l}\text { Origen del Consejo Supremo de España } \\
\text { en Viena }\end{array}$ & 1992 & 5 & 3 \\
\hline Martínez Millán, José & $\begin{array}{l}\text { Las élites de poder durante el reinado de } \\
\text { Carlos V a través de los miembros del } \\
\text { Consejo de Inquisición (1516-1558) }\end{array}$ & 1988 & 5 & 4 \\
\hline Cepeda Adán, José & $\begin{array}{l}\text { Desamortización de tierras de las Órde- } \\
\text { nes Militares en el reinado de Carlos I }\end{array}$ & 1980 & 4 & 0 \\
\hline $\begin{array}{l}\text { Gutiérrez Nieto, Juan } \\
\text { Ignacio }\end{array}$ & $\begin{array}{l}\text { Estructura castizo-estamental de la } \\
\text { sociedad castellana del siglo XVI }\end{array}$ & 1973 & 4 & 0 \\
\hline $\begin{array}{l}\text { Gutiérrez Nieto, Juan } \\
\text { Ignacio }\end{array}$ & $\begin{array}{l}\text { Semántica del término Comunidad antes } \\
\text { de 1520: las asociaciones juramentadas } \\
\text { de defensa }\end{array}$ & 1977 & 4 & 0 \\
\hline $\begin{array}{l}\text { Luxán Meléndez, } \\
\text { Santiago de. }\end{array}$ & $\begin{array}{l}\text { Contribución al estudio de los presidios } \\
\text { españoles del Norte de África. Las difi- } \\
\text { cultades de la plaza de Ceuta para abas- } \\
\text { tecerse de trigo (1640-1668) }\end{array}$ & 1975 & 4 & 0 \\
\hline Mercader Riba, Juan & $\begin{array}{l}\text { La ordenación de Cataluña por Felipe V: } \\
\text { La Nueva Planta. }\end{array}$ & 1951 & 4 & 0 \\
\hline Ruiz Martín, Felipe & $\begin{array}{l}\text { Las finanzas españolas durante el reina- } \\
\text { do de Felipe II }\end{array}$ & 1968 & 4 & 0 \\
\hline Belenguer Cebría, Ernest & $\begin{array}{l}\text { La problemática del cambio político en } \\
\text { la España de Felipe II. Puntualizaciones } \\
\text { sobre su cronología }\end{array}$ & 1980 & 4 & 1 \\
\hline Carrasco, R. & $\begin{array}{l}\text { Preludio al "Siglo de los portugueses.". } \\
\text { La Inquisición de Cuenca y los judaizan- } \\
\text { tes lusitanos en el siglo XVI }\end{array}$ & 1987 & 4 & 1 \\
\hline $\begin{array}{l}\text { Ladero Quesada, Miguel } \\
\text { Ángel }\end{array}$ & $\begin{array}{l}\text { La esclavitud por guerra a fines del siglo } \\
\text { XV: el caso de Málaga. }\end{array}$ & 1967 & 4 & 1 \\
\hline $\begin{array}{l}\text { Álvarez-Coca González, } \\
\text { María Jesús }\end{array}$ & $\begin{array}{l}\text { La Corona de Aragón: Documentación } \\
\text { en el Consejo y la Cámara de Castilla } \\
(1707-1834) \text {. Fuentes del Archivo } \\
\text { Histórico Nacional }\end{array}$ & 1989 & 4 & 2 \\
\hline
\end{tabular}

Hispania, LXII/1, núm. 210 (2002) 41-64 
Uno de los aspectos interesantes a la hora de realizar el estudio es conocer qué artículos publicados en Hispania han tenido mayor impacto entre la comunidad de investigadores de Historia Moderna. Para ello se recogieron los trabajos que habían sido citados mayor número de veces distinguiendo, además, cuántas de estas citas eran autocitas (un autor que citaba un artículo suyo publicado en Hispania desde otra revista).

Se encontró un total de 17 artículos, cada uno de los cuales recibió 4 o más citas. El trabajo más citado era el de Henry Kamen: «El establecimiento de los intendentes en la administración española», que fue citado por ocho autores diferentes durante el período estudiado. El artículo de Miguel Ángel Ladero Quesada: «La repoblación del reino de Granada anterior al año 1500» recibió un total de 7 citas, mientras que el publicado por Manuel Martín Galán: «Fuentes y métodos para el estudio de la Demografía Histórica castellana durante la Edad Moderna» fue citado por 6 autores diferentes.

Aun cuando algunos trabajos como el de Miguel Ángel Echevarría Bacigalupe, Virginia León Sanz o José Martínez Millán habían recibido 5 citas, la mayoría de ellas fueron realizadas por los propios autores. Hay que tener en cuenta, no obstante, que estos artículos han sido innovadores o aclaratorios sobre las temáticas abarcadas convirtiéndose, en ocasiones, en obligada referencia para posteriores trabajos.

TABLA 17. Distribución de los artículos más citados en el período estudiado

\begin{tabular}{|l|c|}
\hline DÉCADAS & N $^{\circ}$ ART. MÁS CITADOS \\
\hline $1940-1949$ & - \\
\hline $1950-1959$ & 1 \\
\hline $1960-1969$ & 4 \\
\hline $1970-1979$ & 3 \\
\hline $1980-1989$ & 7 \\
\hline $1990-1998$ & 2 \\
\hline TOTAL: & 17 \\
\hline
\end{tabular}

La década de los años 80 fue en la que se publicó el mayor número de artículos más citados entre la comunidad de investigadores. Durante los primeros 10 años de existencia de la revista no se publicó ninguno de los artículos mas citados.

\subsection{Antigüedad de los trabajos citados}

La antigüedad de los trabajos citados nos permite conocer el tiempo transcurrido desde que se publican hasta que son citados. 
FRANCISCO TOSETE HERRANZ

TABLA 18. Antigüedad de los trabajos citados

\begin{tabular}{|l|c|c|c|}
\hline ANTIGÜEDAD & $\begin{array}{c}\mathbf{N}^{0} \text { DE CITAS HECHAS A TRABAJOS } \\
\text { DE HISPANIA }\end{array}$ & $\%$ & $\%$ ACUM. \\
\hline de 0 a 9 años & 128 & 34,78 & 34,78 \\
de 10 a 19 años & 75 & 20,38 & 55,16 \\
de 20 a 29 años & 75 & 20,38 & 75,54 \\
de 30 a 39 años & 50 & 13,59 & 89,13 \\
de 40 a 49 años & 37 & 10,05 & 99,18 \\
de 50 a 59 años & 3 & 0,82 & 100 \\
\hline Total: & 368 & 100 & \\
\hline
\end{tabular}

Las tres cuartas partes de los trabajos citados $(75,54 \%)$ tenían 29 años o menos de antigüedad. La vida media o el tiempo durante el cual fue publicada la mitad de los trabajos de Historia Moderna de Hispania se situaba en los 17,5 años. Este período de tiempo, no excesivamente elevado para éste área, ponía de relieve cómo eran más citados los trabajos actuales que los publicados durante los casi cincuenta años transcurridos desde la aparición de Hispania.

\section{CONCLUSIONES}

En el conjunto de revistas analizadas en el presente estudio se publicaron un total de 1279 trabajos (artículos, estudios críticos, notas bibliográficas, ensayos, bibliografía histórica, textos y documentos o miscelánea) relativos al área de Historia Moderna en los últimos 10 años.

Desde su inicio en 1940 se publicaron en Hispania 434 artículos de Historia Moderna, 61 de los cuales aparecieron entre 1967 y 1983 en los Cuadernos de Historia. Anexos de Hispania.

Del total de las citas encontradas se observó cómo una gran parte $(92,39 \%)$ correspondía a trabajos aparecidos en la sección estudios/artículos de Hispania que se perfilaba como la de mayor importancia y contenido científico de la revista.

En los artículos citados las temáticas más representativas eran las relacionadas con las instituciones. Casi una quinta parte de los mismos $(19,12 \%)$ trataba sobre esta materia. En un porcentaje algo menor, en torno a un $17 \%$, se situaban los artículos que versaban sobre los grupos sociales y la mentalidad, o la economía, respectivamente, y con algo más del $15 \%$ los relativos a la demografía, población y alimentación. Las temáticas restantes eran mucho menos significativas.

Más de la mitad de los artículos de Historia Moderna publicados en Hispania , habían sido citados en los últimos diez años en alguna de las revistas analizadas $(52,28 \%)$. Asimismo, de los artículos de Cuadernos de Historia. Anexos de

Hispania, LXII/1, núm. 210 (2002) 41-64 
Hispania se citaron algo más de la cuarta parte (26,23\%). Por término medio, cada artículo de Historia Moderna aparecido en la revista Hispania recibió 1,63 citas, frente a los publicados en los Cuadernos de Historia Moderna. Anexos de Hispania que recibieron 1,37 citas.

En los últimos 20 años se observaba una disminución del número de artículos de Historia Moderna citados respecto de los publicados en Hispania. Esta disminución venía ocasionada por la aparición de nuevas revistas especializadas de Historia Moderna en el ámbito universitario. Asimismo, en los últimos nueve años la disminución era particularmente acusada (un 21,8\% menos respecto de la década anterior) debido, entre otras razones, al mayor número de artículos de Moderna publicados en dicho período en la revista y al escaso tiempo transcurrido desde su aparición, dado que el tiempo medio que había de transcurrir desde que un artículo era publicado en Hispania hasta que se citaba era de 17,5 años.

De las revistas analizadas Cuadernos de Historia Moderna era, con gran diferencia, aquella desde la que se realizaban mayor número de citas a trabajos de Hispania (31.09\%) seguida de Studia Historica, de donde provenían casi la quinta parte (18,49\%) y en menor medida Chronica Nova (14,29\%), Manuscrits $(11,76 \%)$ y Pedralbes $(9,24 \%)$. En el resto de revistas el porcentaje de citas a trabajos de Hispania era inferior al $6 \%$.

De las citas totales recogidas, realizadas a trabajos de Historia Moderna publicados en Hispania y Anexos, una gran parte $(43,48 \%)$ correspondía a artículos aparecidos en los últimos 20 años. En las cuatro últimas décadas el porcentaje de artículos citados publicados en la revista se mantenía constante, en torno al $20 \%$ cada década, de las citas totales.

El nivel de autocitación de los autores era relativamente bajo situándose en algo menos del $7 \%$ en el caso de Hispania, e inferior al $11 \%$ en el resto de las revistas analizadas. Se observaba, además, cómo la mayor parte de los autores no trabajaban en colaboración (casi un $90 \%$ publicaba en solitario). Ninguno de los artículos citados había sido publicado por más de tres autores.

El $66 \%$ de los autores tan sólo habían sido citados una vez, y algo menos del $15 \%$ habían recibido 4 o más citas. Por otro lado, de los 20 autores más citados el $75 \%$ trabajaba en centros u organismos de Madrid mientras que el resto desempeñaba su actividad en diversos centros españoles.

Sorprendía el hecho de que 6 de ellos (30\%) no eran propiamente especialistas de Historia Moderna sino de otras áreas como Historia Medieval, Historia Contemporánea, Historia de la Ciencia y Archivística.

Puede concluirse cómo la aparición de nuevas revistas especializadas en Historia Moderna ha influido en la disminución del impacto que Hispania tiene en la comunidad de investigadores modernistas. No hay que olvidar que Hispania es una revista generalista. Aún así, dado que la Historia Moderna es el área temática que mayor representación tiene en ella, parece necesario actuar con el fin de corregir el desequilibrio detectado en el número de artículos citados de los últimos años. 
GrÁFICO 1 (Tabla 4)

\section{Tipología de los trabajos de Hispania citados}

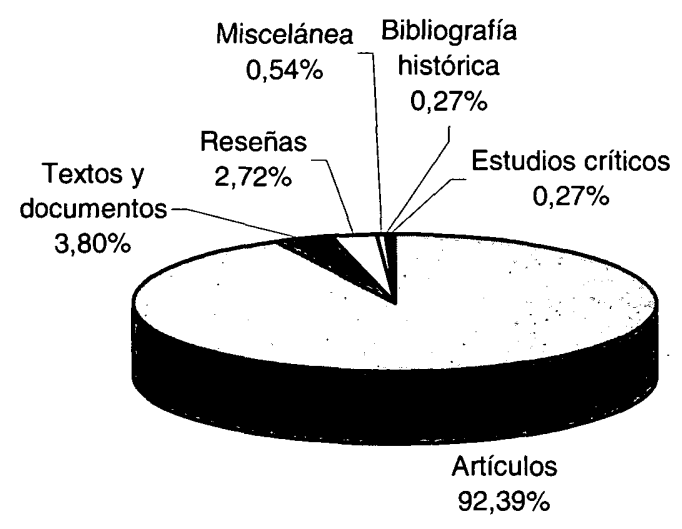

GRÁfICO 2 (Tabla 5)

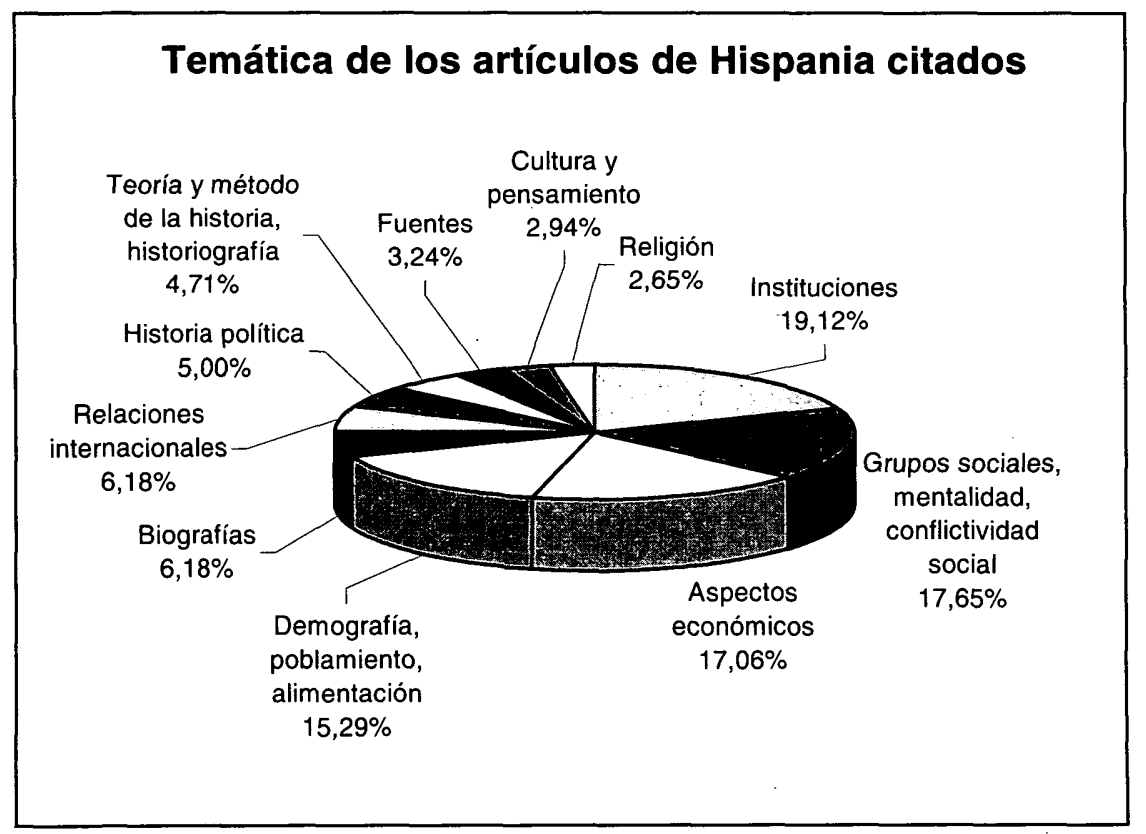

Hispania, LXII/1, núm. 210 (2002) 41-64 
GRÁFICO 3 (Tabla 6)

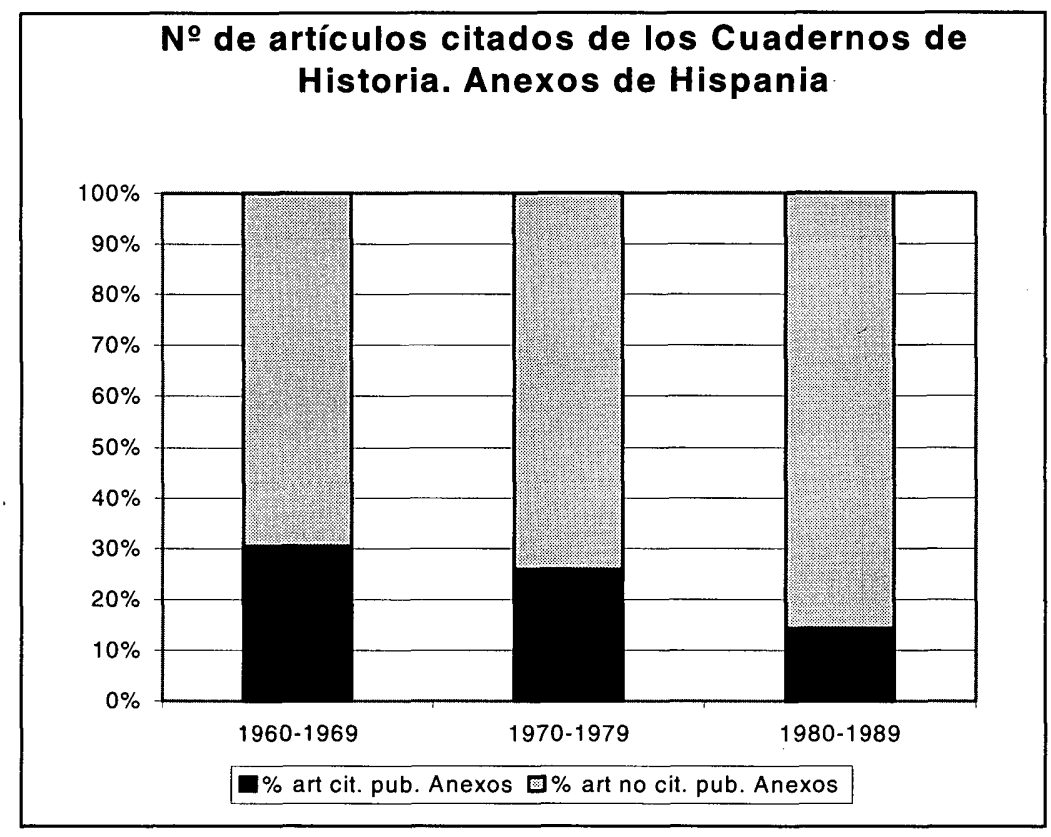

GRÁFICO 4 (Tabla 7)

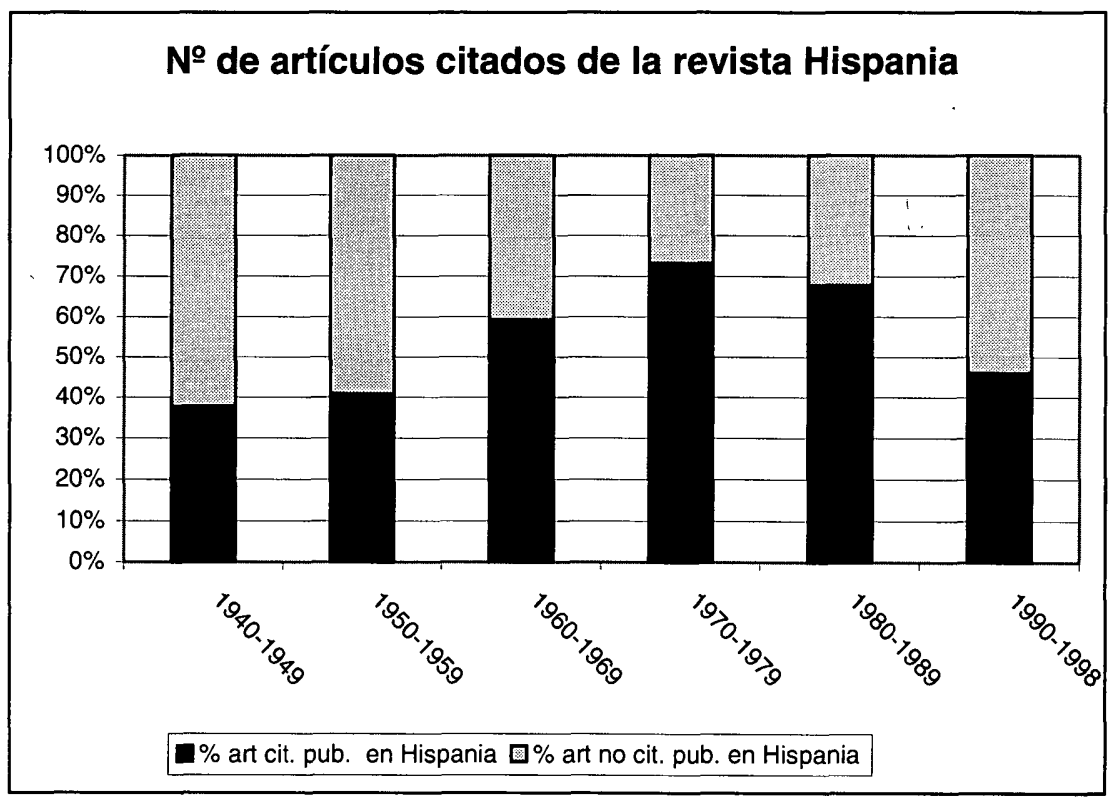

Hispania, LXII/1, núm. 210 (2002) 41-64 


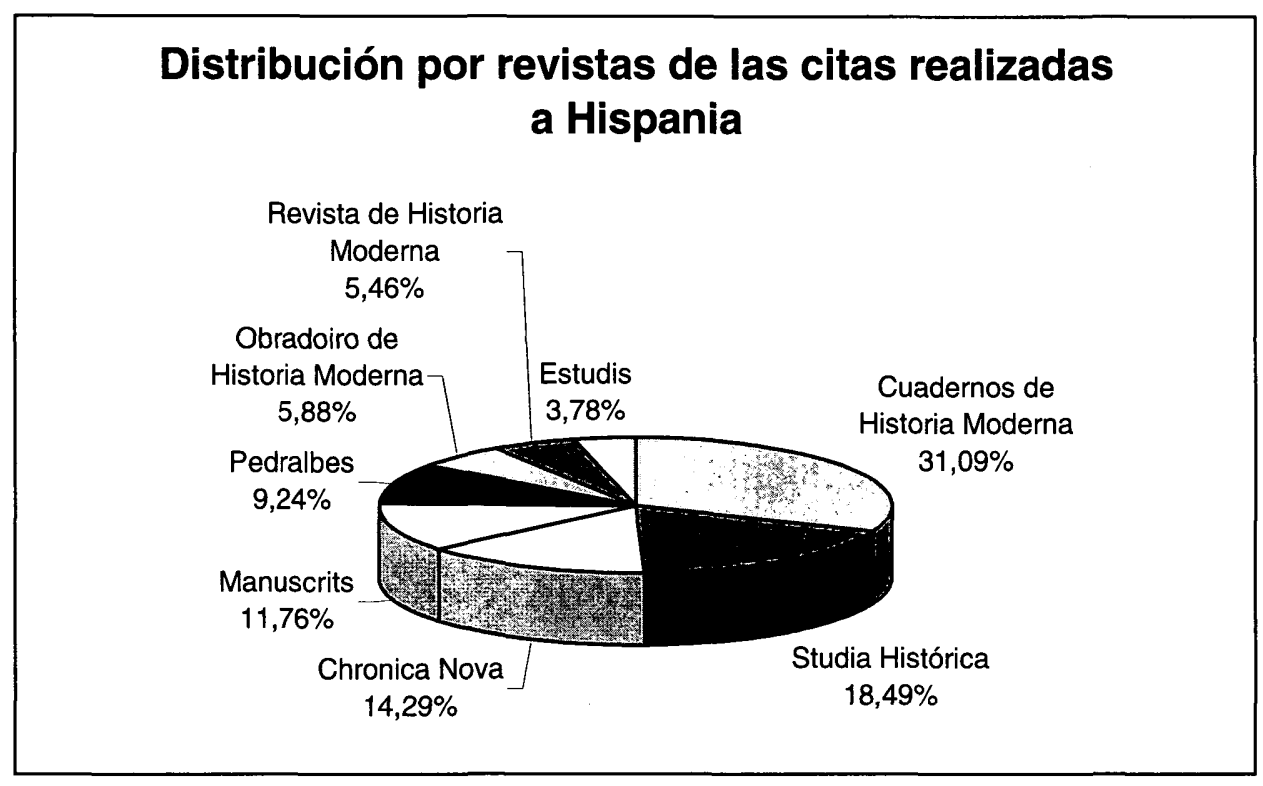

GRÁFICO 6 (Tabla 10)

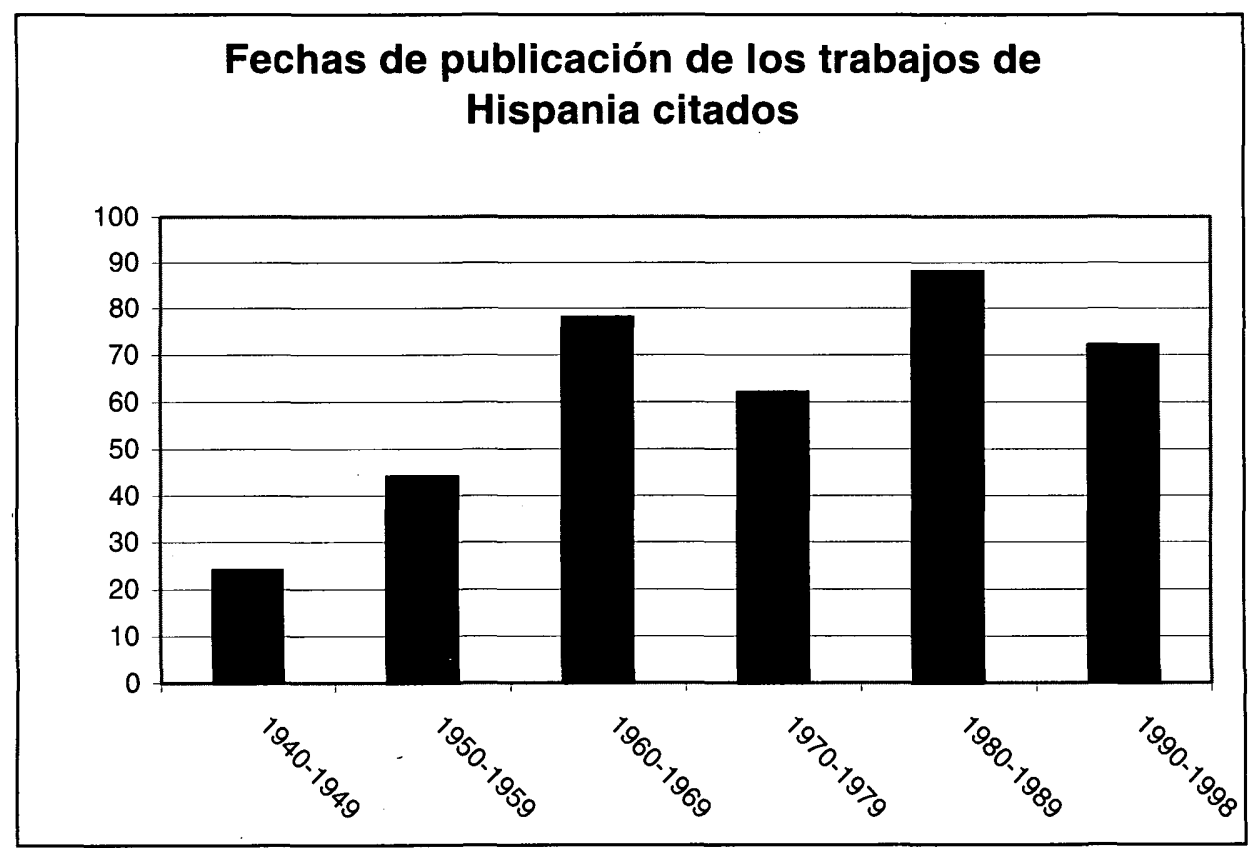

Hispania, LXII/1, núm. 210 (2002) 41-64 


\section{BIBLIOGRAFÍA}

ALmuiña FernánDeZ, C. et. al.: Hispania, revista de Historia (1940-1989). Análisis y evolución de contenidos. Hispania. 1990 , vol. L/2, $\mathrm{n}^{\circ} 175$, p. 393-416

CARRASCAL, L.M.; DiAZ, M.: Utilidad científica y difusión internacional de Ardeola: un análisis bibliométrico. [en línea]. Ardeola. 1998, $\mathrm{n}^{\circ}$ 45. «http://www.mncn.csic.es/ investigacion/carrascal/ardeola/ardeola.html» [Consulta: 15 junio 2000]

CRUZ Rubio LINIERS, M.; ROSARIO RUIZ FRANCO, M ${ }^{\mathrm{a}}$. DEL.: La investigación histórica sobre el franquismo: un análisis bibliométrico de las revistas españolas. Hispania. 1994 , vol. LIV/2, n 187 , p. 661-676

EsTEPA, C.: Las revistas de Historia de España: el ejemplo de Hispania. Revista de Historia Jerónimo Zurita.1995 n 71, p.297-308

FRÍAS, J.A.; ROMERO GÓMEZ, P.: ¿Quiénes son y qué citan los investigadores que publican en las revistas españolas de Biblioteconomía y Documentación?. [en línea]. Anales de Documentación. 1998, vol. 1. «http://www.um.es/fccd/anales/ad01/ad0104.html» [Consulta: 1 julio 1999]

SÁNCHEZ MARCOS, F.; PÉREZ LATRE, M.: La historiografía de la época moderna (14741808) en la revista «Hispania», 1940-1988. Hispania. 1990, vol. L/3, n 176, p. 1031-1045

SANZ, E.; García Zorita, C.; García Romero, A.; Modrejo Rico, A.: La investigación española en economía a través de las publicaciones nacionales e internacionales en el período 1990-1995. Revista de Economía Aplicada. 1999, vol. VII, n² 20, p. 113-137

SANZ, E.; ARAGÓN, I.; MÉNDEZ, A.: ¿Cumplen algún papel las revistas científicas nacionales?. Boletín de la Sociedad Española de Cerámica y Vidrio. 1996, vol. 35, n 1 , p. 35-40

SANZ, E.; ARAGÓN, I.; MÉNDEZ, A.: The function of national journals in disseminating applied science. Journal of Information Science. 1995, vol. 21, $\mathrm{n}^{\circ} 4$, p. 319-323

VÁZQuez VAlero, M.; MORIllo ARIZA, F.; GómeZ CARIDAD, I.: Estudio de la revista «Boletín de la Sociedad Española de Cerámica y Vidrio». Boletín de la Sociedad Española de Cerámica y Vidrio. 1999, vol. 1, no 38, p. 35-49 